\title{
Current controversies in the cognitive science of short-term memory
}

\begin{abstract}
Short-term memory is critically implicated in most human cognitive capacities and has been the object of study for more than a century, yet many questions remain unsettled and new controversies have emerged. This paper provides an overview of some current debates within the field. These include (i) the issue of how many short-term memory systems there are, (ii) whether working memory is best understood as having domain-specific resources, (iii) how information is encoded in working memory, (iv) how sensory memory and working memory are related to attention, and (v) the relationship between short-term memory and consciousness.
\end{abstract}

Memory, in the popular sense of the term, is associated with our capacity for recalling events minutes, hours, or years in the past. However, to use information we must represent or store it somewhere in our minds, even if only fleetingly. Hence some form of short-term memory is involved in capacities such as recognising an object [1], reading a text [2], or detecting changes in presented information [3].

Despite more than a century of research into the different forms of short-term memory, a number of major unsettled questions remain. This brief review will examine some of the most pressing current debates, with particular focus on those relevant to theoretical debates in cognitive science. As a result, it will largely pass over other valuable discussions concerning, for example, memory and intelligence and issues relating to development and aging.

\section{How many short-term memory systems?}

The dominant historical approach to short-term memory has involved positing different shortterm memory systems. Atkinson and Shiffrin famously drew a distinction between sensory memory and short-term memory proper. While this model is now recognised to be overly simplistic, it has served as a basis for more nuanced frameworks for carving up the space of short-term memory systems.

Most contemporary memory researchers recognise at the very least a distinction between the various forms of sensory memory - such as iconic memory [4] and echoic memory [5] - and working memory [6] (however, see [7] for a dissenting view). The status of other posited memory systems is more controversial. There is some evidence, for example, for a form of visual sensory memory with somewhat smaller capacity but greater duration (up to several seconds) than iconic memory dubbed Fragile Visual Short-Term Memory (fVSTM) [8]). Some have suggested that this apparent new store can be explained away via a more complex account of differences in attentional processing of items in iconic memory [9], while others 
have suggested that fVSTM and sensory memory should be considered parts of an integrated system of 'perceptual memory' [10].

A second recently mooted memory store is Conceptual Short-Term Memory (CSTM) $[11,12]$. CSTM is invoked to explain our ability to seemingly effortlessly understand the conceptual significance of perceptual stimuli. It is conjectured to have a larger capacity but shorter duration than working memory, while differing from iconic memory insofar as it encodes not merely sensory but semantic features of objects. Evidence for CSTM comes from a variety of experiments, perhaps most notably Rapid Serial Visual Presentation (RSVP) tasks that show subjects can retain basic gist information of up to twelve very rapidly serially presented stimuli [13]. If true, these results would suggest (in light of the very brief presentation times of as little as $13 \mathrm{~ms}$ per image) that feedforward processes alone can result in conceptualisation of visually presented information, a conclusion that remains controversial $[14,15]$.

\section{The structure of working memory}

In addition to debates about the number of basic short-term memory systems, there are longstanding questions concerning the number of subsystems present in working memory specifically. An influential account of working memory due to Baddeley and Hitch [16] posits a multi-component store in which visuospatial and auditory-verbal information are retained in separate buffers of a single system. This model is supported by evidence that working memory tasks in separate modalities do not generate interference [17].

An alternative approach sees working memory as a central capacity-limited mechanism shared across multiple sensory modalities [18]. Some recent evidence for this alternative proposal comes from a review suggesting that patients displaying working memory deficits for one kind of information typically possess deficits in other domains and sensory modalities [24]. Moreover, one experimental assessment of between-domain interference in different working memory domains found that while a probe recognition task was not subject to crossdomain interference, there was such interference in the recall of verbal and visuospatial information [21].

Complicating matters, however, and contradicting earlier results [22], multiple findings now suggest that working memory training in one domain does not exhibit 'far transfer' effects to other domains [23]. Hence despite the apparently straightforward nature of the question as to 
whether or not working memory involves separate non-interfering components, the matter remains frustratingly contested.

A related controversy concerns whether representations in working memory are simply those portions of working memory activated by a "focus of attention" or instead constitute separate copies held in a dedicated short-term store (see Fig. 1). Put another way, we might ask whether short-term memory stores information or instead simply stores pointers to information encoded elsewhere.

A

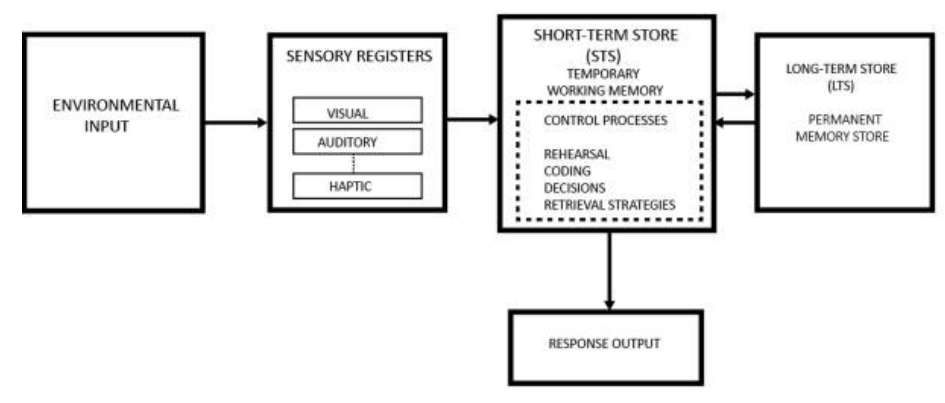

B

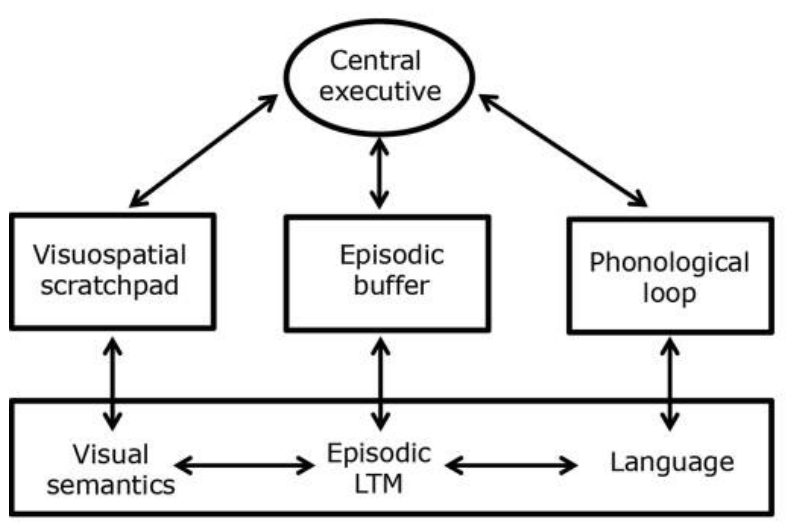

C

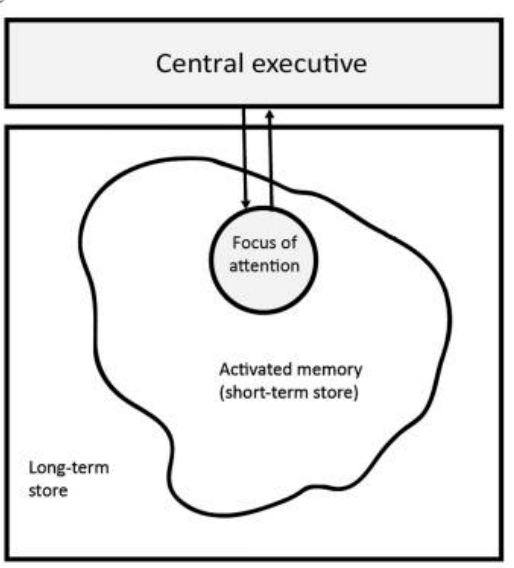

D

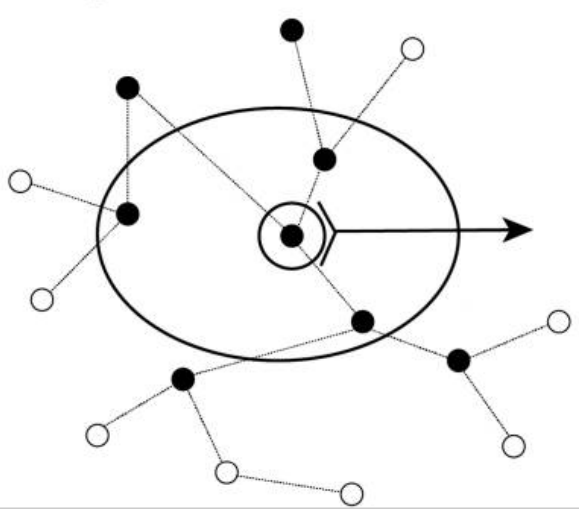

Fig. 1- a comparison of different models of long- and short-term memory. (A) and (B) depict separate store models, respectively those of Atkinson \& Shiffrin [24] and Baddeley \& Hitch [6]. (C) depicts the model of Cowan [25] in which information in long-term memory is activated via attentional processes. (D) depicts the related model of Oberauer [26] in which lines and circle represent nodes in long term memory, with black nodes indicating activated portions. The larger oval represents the 'direct access region' and the smaller circle represents the focus of attention. From Norris [27].

While some neuroimaging data has been brought to bear on the question, many recent moves in this debate have focused part on theoretical rather than experimental considerations. For example, while the activated long-term memory (aLTM) view may have the advantage of 
allowing for more efficient encoding of information, it has been questioned whether it can adequately capture our ability to recall wholly novel or information and to recall information about the order in which stimuli are presented [27]. Nelson Cowan in defending the aLTM view suggests that information can be encoded rapidly in LTM, and that sequence information can be represented via a structured system of pointers [25], a move that one critic has claimed is "simply an exercise in relabelling" [28]. As in the case of the debate concerning whether working memory involves a single domain general or multiple domainspecific stores, the question of the relationship between long-term memory and working memory remains unresolved.

\section{How many slots in working memory?}

One robust finding in the science of short-term memory has been the observation that humans are strikingly limited in the number of discrete items that can be recalled from a given dataset when appropriate constraints are in place to safeguard against the use of mnemonic devices and rehearsal. In one of the most widely cited papers in all of psychology, George Miller estimated a limit of around "seven, plus or minus two" [29], though later work reduced this to around 3-5 items [30].

The idea that working memory involves a discrete number of slots has however been challenged by the idea that working memory involves a continuous 'mnemonic resource' that can be allocated variably to different items in accordance with task demands [31,32]. Evidence for this model comes from multiple experiments whose results are seemingly at odds with earlier slot-based approaches. For example, in one experiment subjects were presented with an array of coloured squares and after stimulus removal were tasked with matching the colour of a probed item using a colour wheel (see Fig.2). According to a slotsbased approach, one might expect a rapid dropoff in performance once the number of items in the initially presented array exceeded the number of slots in working memory. What was found, however, was a continuous decline in performance proportionate to the number of items presented. 

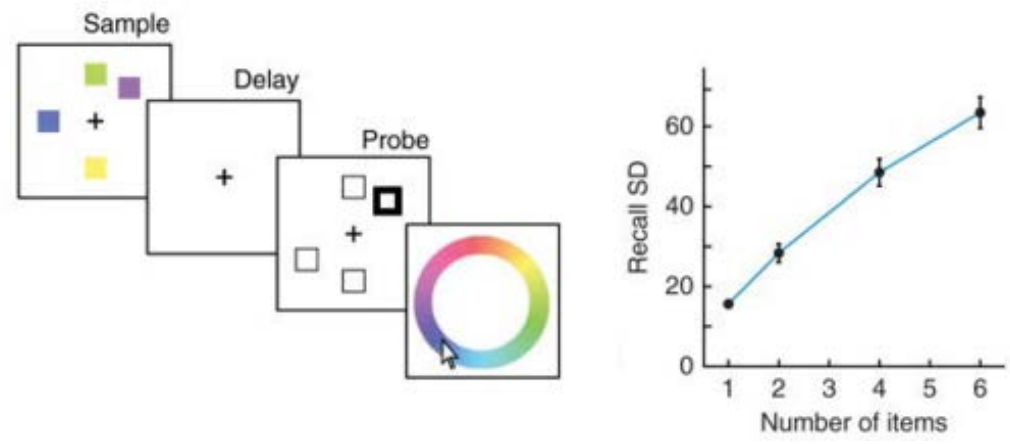

Fig. 2. A colour delayed-estimation task (from Ma et al. [31], summarizing [33]). Subjects are presented with variable numbers of coloured squares and given a cue following a short delay. The task was to indicate via a colour wheel the exact colour of the square that had been cued. Subject's accuracy as indicated by recall variability decreased smoothly in proportion to the quantity of squares in the initial stimulus. This conflicts with predictions of a slot-based model, which might be expected to anticipate smooth performance up to around four items followed by a rapid degrading thereafter.

In light of experiments such as these [34], a view has emerged even among prior defenders of a purely slot-based approach that a more fine-grained approach to the retention and encoding of information in working memory is required [43; however, see 44]. Correspondingly, greater emphasis is now placed by many prominent memory researchers on computational and information-theoretic measures of memory. Rather than a simple picture of activating and encoding information in discrete slots, then, many researchers now recognise that shortterm memory should be understood in terms of the demands of extracting signals from noisy data by selective and continuous attentional amplification and restoration of information $[37,38]$.

\section{Short-term memory and attention}

The relationship between short-term memory and attention is also highly vexed, no doubt in part because of the wide variety of forms of attention and the various functions it might play in the encoding, retention, and access of information in memory [39], as well as the still unresolved controversies about the architecture of memory already discussed. A detailed review of the relevant literature is beyond the scope of the present paper, but a few notable results can be reported.

First, it has long been recognised that attention in some sense facilitates working memory, but its exact role in unclear. Thus the initiation of the consolidation of visually presented information into working memory seems to require attention [40]. On the assumption that attention is in some sense is a limited-capacity mechanism or 'bottleneck' in processing, this 
would explain the phenomenon of the 'attentional blink' in which subjects often fail to see a stimulus presented immediately after a target [41]. By contrast, evidence for a role for attention in the maintenance of previously presented information is much more contested. For example, as children enter adolescence, their ability to manage distinct stores information relevant to different tasks seems to increase, despite no concomitant improvement in core attentional capabilities [42]. Evidence such as this has led some to suggest that information once consolidated into working memory can be "offloaded", allowing maintenance to occur without the need for the allocation of attention [43]. An alternate influential model known as time-based resource sharing holds the role of attention in maintenance to involve not continual retention of individual items but rather the periodic and serial 'refreshing' of items to prevent decay [44].

A second ongoing debate concerns the relationship between perceptual attention and memory-directed attention. One reason to think these mechanisms are closely linked comes from the finding that many of the properties of objects that facilitate or impair the selection of items in perceptual tasks - such as target-distractor similarity effects - also do so for working-memory tasks [52; see also 53]. Likewise, there is some reason to think that performance in tasks that place heavy demands on perceptual attention is related to measures of cognitive performance, specifically fluid IQ [47]. This is somewhat supported by neuroimaging results that show considerable overlap in brain regions recruited for visual and memory-based forms of attention [48]. Evidence for interference between cognitive load and perceptual performance (and vice-versa) however is more mixed. For example, while there is evidence that cognitive load may influence even early perceptual processing [49], it seems to do so in ways quite different from perceptual load. One study using realistic advertisements placed around a browser game, for example, found that perceptual load reduced recognition of the adverts, while cognitive load made participants more likely to notice them [50], a finding supported by a similar experiment involving distraction by billboards while driving [51]. One possible explanation is that perceptual attention is best understood as involving distinct mechanisms for spatial tracking and feature tracking [57].

A final debate worth briefly noting concerns the relationship between attention and sensory memory. While it has long been assumed that sensory memory operates effortlessly and thus does not require attention, some doubt has been cast on this by recent findings. In one experiment, subjects were shown either letter-matrices or coloured circles and instructed to perform either a letter recall task or a colour-circle task respectively. After initial separate training on these two tasks, the displays were combined with subjects performing one or 
another task as instructed by a cue. The striking finding of the experiment was that subjects entirely failed to notice when in one colour-circle cue the background letter display was absent entirely, leading them to conclude that inattention prevents the encoding of sensory information in iconic memory [54]. While these findings are not uncontested [55], they highlight an intriguing and underexplored area for research on short-term memory and attention.

\section{Short-term memory and consciousness}

A final vexed issue in the philosophy and science of short-term memory research concerns its relationship to consciousness. Two particular ongoing discussions are worth noting. The first is the so-called 'overflow' debate developed by philosopher Ned Block, and it concerns whether the contents of sensory memory might themselves be conscious independent of encoding in working memory [56]. Multiple experiments spanning several decades have indicated that items in iconic memory can be recruited via postcues for the performance of tasks, with subjects having an impression of having seen these items all along and thus (perhaps) consciously perceived them. More recent work has provided further evidence to this effect, with the finding that subjects are able to access information about the diversity of colours in an array via a postcue [57]. Interpretation of the results is complex, to say the least, with some suggesting, for example, that subjects' impression of 'phenomenal richness' can be better explained via an illusory process of interpretation subsequent to cueing [58].

Other support for the overflow hypothesis has come from the use of 'no-report paradigms' [59]. These involve trials that do not require subjects to make reports of their experience, but instead use third-personal measures such as eye-movement monitoring during binocular rivalry combined with neuroimaging to track changes in neural activation during changes in conscious experience. In principle, these might allow us to identify and compensate for the specific contributions to neural activation in perceptual tasks made by requirements of report. Some results of this kind have suggested a far smaller role than traditionally assumed for prefrontal regions involved in executive control and working memory in comparison with occipital areas associated with sensory processing. Pushback to such claims has come from authors who suggest, for example, that no-report paradigms introduce distinctive confounds of their own [59], and should not overrule existing evidence for a critical role for the prefrontal cortex in conscious perception [60].

A second debate has arisen concerning whether working memory itself is always conscious. While it has long been assumed that working memory "should contain what we think of as 
the conscious mind" [35], recent experiments have put this in doubt. For example, it has been demonstrated that masked and unreportable Gabor patches can still be used after a delay of several seconds for the purposes of an orientation-matching task [61]. In light of these relatively long delays and the use of pattern masks, an explanation for this capacity in terms of sensory memory seems unlikely, while a working memory interpretation is supported by the presence of accompanying signal changes in the prefrontal cortex [62]. Against this interpretation, some have suggested the possibility of accompanying conscious experience below the threshold required for confident report [63], as well as the worry that, absent evidence for active maintenance of the relevant information, so-called unconscious working memory might merely involve a priming effect that facilitated subsequent guessing [64].

\section{Conclusion}

It is easy to feel despondent about the state of short-term memory research: how can we have so much evidence yet so few firm conclusions? In light of this, there are grounds for thinking that a move away from a narrow systems-based approach to short-term memory may offer at the very least some new avenues of investigation, whether in the form of active inference frameworks [65], computational approaches [66], or simply better theory-neutral benchmarks for assessing competing models [67]. Likewise, while neuroscientific data has long played an important role in informing and constraining theoretical debates about short-term memory, one might hope that by identifying or modelling specific memory mechanisms - such as the oscillatory properties of neurons encoding working memory information [68] - new light may be shed on longstanding debates, especially in light of ongoing progress in neuroimaging techniques. Broader progress in neuroscience towards understanding the biological basis of different cognitive capacities may also make important contributions to our understanding of short-term memory, as demonstrated for example by the growing interest in the contribution of white matter to working memory function [69].

On a separate note, there may be grounds for paying closer attention to cultural differences in constructing models of short-term memory. In one important recent study [70], subjects' spatialization of even non-verbal information in working memory was shown to be heavily influenced by language and literacy, with literate Arabic speakers encoding information about sequentially presented Gabor patches in a right-to-left direction, in contrast both to Western readers (who encoded information in a left-to-right direction) and illiterate Arabic speakers (who showed no systematic spatial organisation).

As a final counsel against despair, we should not forget that cognitive science is a relatively young field and one that present immense challenges. Aside from a few spectacular 
successes in fields such as early visual processing, the norm of the field has been tentative progress dogged by continued debate about concepts and methods. Rather than anticipating that answers will emerge spontaneously from data, then, we should expect insights to emerge in fits and starts from a background of lively theoretical disagreement.

\section{ACKNOWLEDGEMENTS}

This work was supported by the Leverhulme Centre for the Future of Intelligence, Leverhulme Trust, under Grant RC-2015-067.

\section{REFERENCES}

1. Ricker TJ, Thiele JE, Swagman AR, Rouder JN: Recognition Decisions From Visual Working Memory Are Mediated by Continuous Latent Strengths. Cogn Sci 2017, 41:1510-1532.

2. Peng P, Barnes M, Wang CC, Wang W, Li S, Swanson HL, Dardick W, Tao S: Metaanalysis on the relation between reading and working memory. Psychol Bull 2018, 144:48-76.

3. Leising K, Jacqmain J, Elliott C, Wolf J, Taylor J, Cleland L, Lee R, Magnotti JF, Wright AA: Sensory and Working Memory in a Spatial Change-Detection Task by Pigeons and Humans. Behav Processes 2019, doi:10.1016/j.beproc.2019.103957.

4. Sugita Y, Hidaka S, Teramoto W: Visual percepts modify iconic memory in humans. Sci Rep 2018, 8.

5. Kinukawa T, Takeuchi N, Sugiyama S, Nishihara M, Nishiwaki K, Inui K: Properties of echoic memory revealed by auditory-evoked magnetic fields. Sci Rep 2019, 9.

6. Baddeley AD, Hitch G: Working memory. In Exploring Working Memory. . Routledge; 2017:43-79.

7. Gross S, Flombaum J: Does Perceptual Consciousness Overflow Cognitive Access? The Challenge from Probabilistic, Hierarchical Processes. Mind Lang 2017, 32:358-391.

8. Vandenbroucke ARE, Sligte IG, De Vries JG, Cohen MX, Lamme VAF: Neural correlates of visual short-term memory dissociate between fragile and working memory representations. J Cogn Neurosci 2015, 27:2477-2490.

*9. Robinson MM, Irwin DE: Are there two visual short-term memory stores? A statetrace analysis. $J$ Math Psychol 2019, 90:23-30.

This paper is a response to [22] and constitutes an important recent salvo in the debate concerning whether working memory involves domain-specific or domain general mechanisms. The paper replicated the experimental procedure of [22] involving concurrent maintenance of visual-spatial and auditory verbal information and showed that there was no dual-task cost associated with a probe recognition task. However, it found substantial between-domain interference in the recall of visuospatial information. 
10. Yatziv T, Kessler Y: A two-level hierarchical framework of visual short-term memory. $J$ Vis 2018, 18:1-26.

11. Potter MC: The immediacy of conceptual processing. In On Concepts, Modules, and Language: Cognitive Science at its Core. . Oxford University Press; 2017:239-248.

12. Shevlin H: Conceptual short-term memory: A missing part of the mind? $J$ Conscious Stud 2017, 24:163-188.

13. Potter MC, Wyble B, Hagmann CE, McCourt ES: Detecting meaning in RSVP at 13 ms per picture. Attention, Perception, Psychophys 2014, 76:270-279.

14. Marti S, Dehaene S: Discrete and continuous mechanisms of temporal selection in rapid visual streams. Nat Commun 2017, 8.

15. Howe PDL: Natural scenes can be identified as rapidly as individual features. Attention, Perception, Psychophys 2017, 79:1674-1681.

16. Baddeley AD, Hitch G: Working Memory. 1974:47-89.

17. Fougnie D, Zughni S, Godwin D, Marois R: Working memory storage is intrinsically domain specific. J Exp Psychol Gen 2015, 144:30-47.

18. Cowan N: The magical mystery four: How is working memory capacity limited, and why? Curr Dir Psychol Sci 2010, 19:51-57.

19. Morey CC: The case against specialized visual-spatial short-term memory. Psychol Bull 2018, 144:849-883.

20. Peng P, Fuchs D: A Meta-Analysis of Working Memory Deficits in Children With Learning Difficulties: Is There a Difference Between Verbal Domain and Numerical Domain? J Learn Disabil 2016, 49:3-20.

*21. Uittenhove K, Chaabi L, Camos V, Barrouillet P: Is Working Memory Storage Intrinsically Domain- Specific? J Exp Psychol Gen 2019, doi:10.1037/xge0000566.

This paper is a response to [22] and constitutes an important recent salvo in the debate concerning whether working memory involves domain-specific or domain general mechanisms. The paper replicated the experimental procedure of [22] involving concurrent maintenance of visual-spatial and auditory verbal information and showed that there was no dual-task cost associated with a probe recognition task. However, it found substantial between-domain interference in the recall of visuospatial information.

22. Schwarb H, Nail J, Schumacher EH: Working memory training improves visual short-term memory capacity. Psychol Res 2016, 80:128-148.

23. Melby-Lervåg M, Redick TS, Hulme C: Working Memory Training Does Not Improve Performance on Measures of Intelligence or Other Measures of "Far Transfer": Evidence From a Meta-Analytic Review. Perspect Psychol Sci 2016, 11:512-534.

24. Atkinson RC, Shiffrin RM: Human memory: a proposed system and its control processes. Psychol Learn Motiv 1968, 2:89-195.

25. Cowan N: Short-term memory based on activated long-term memory: A review in response to Norris (2017). Psychol Bull 2019, 145:822-847. 
26. Oberauer K: Access to information in working memory: exploring the focus of attention. J Exp Psychol Learn Mem Cogn 2002, 28:411-21.

27. Norris D: Short-term memory and long-term memory are still different. Psychol Bull 2017, 143:992-1009.

*28. Norris D: Even an activated long-term memory system still needs a separate shortterm store: A reply to Cowan (2019). Psychol Bull 2019, 145:848-853.

This paper is highlighted as important not just in isolation but as the most recent work in an ongoing debate between Norris and Cohen concerning whether information in working memory just involves activated portions of long-term memory or instead separate copies (see [34] and [32] for the preceding discussion). In addition to providing a detailed summary of the debate thus far, it provides some interesting new criticisms of Cowan's position.

29. Miller GA: The magical number seven, plus or minus two: some limits on our capacity for processing information. Psychol Rev 1956, 63:81-97.

30. Cowan $\mathrm{N}$ : The magical number $\mathbf{4}$ in short-term memory: A reconsideration of mental storage capacity. Behav Brain Sci 2001, 24:87-114.

31. Ma WJ, Husain M, Bays PM: Changing concepts of working memory. Nat Neurosci 2014, 17:347-356.

32. Bays PM: Spikes not slots: Noise in neural populations limits working memory. Trends Cogn Sci 2015, 19:431-438.

33. Wilken P, Ma WJ: A detection theory account of change detection. J Vis 2004, 4:1120-35.

34. Schneegans S, Bays PM: No fixed item limit in visuospatial working memory. Cortex 2016, 83:181-193.

35. Logie RH, Cowan N: Perspectives on working memory: introduction to the special issue. Mem Cogn 2015, 43:315-324.

36. Adam KCS, Vogel EK, Awh E: Clear evidence for item limits in visual working memory. Cogn Psychol 2017, 97:79-97.

37. Myers NE, Chekroud SR, Stokes MG, Nobre AC: Benefits of flexible prioritization in working memory can arise without costs. J Exp Psychol Hum Percept Perform 2018, 44:398-411.

38. Sprague TC, Ester EF, Serences JT: Restoring Latent Visual Working Memory Representations in Human Cortex. Neuron 2016, 91:694-707.

**39. Oberauer K: Working Memory and Attention - A Conceptual Analysis and Review. J Cogn 2019, 2.

This paper provides a detailed summary of the very latest state of the debate concerning the relationship between working memory and attention. In addition to considering various ways of conceptualising this relationship, it makes several notable claims in its own right (for example, that perceptually attending to items facilitates but does not mandate encoding in working memory), and notes some complications for the view that capacity limits in working memory arise from limitations in attentional resources.

40. Ricker TJ, Nieuwenstein MR, Bayliss DM, Barrouillet P: Working memory 
consolidation: Insights from studies on attention and working memory. Ann $N Y$ Acad Sci 2018, 1424:8-18.

41. Ricker TJ, Hardman KO: The nature of short-term consolidation in visual working memory. J Exp Psychol Gen 2017, 146:1551-1573.

42. Cowan N, Li Y, Glass BA, Scott Saults J: Development of the ability to combine visual and acoustic information in working memory. Dev Sci 2018, 21.

43. Rhodes $\mathrm{S}$, Cowan $\mathrm{N}$ : Attention in working memory: Attention is needed but it yearns to be free. Ann N Y Acad Sci 2018, 1424:52-63.

44. Barrouillet P, Camos V: Essays in cognitive psychology. Working memory: Loss and reconstruction. Psychology Press.; 2015.

45. Kong G, Fougnie D: Visual search within working memory. J Exp Psychol Gen 2019, 148:1688-1700.

46. Kiyonaga A, Egner T: Center-Surround Inhibition in Working Memory. Curr Biol 2016, 26:64-68.

47. Tullo D, Faubert J, Bertone A: The characterization of attention resource capacity and its relationship with fluid reasoning intelligence: A multiple object tracking study. Intelligence 2018, 69:158-168.

48. Myers NE, Stokes MG, Nobre AC: Prioritizing Information during Working Memory: Beyond Sustained Internal Attention. Trends Cogn Sci 2017, 21:449-461.

*49. Liu P, Forte J, Sewell D, Carter O: Cognitive load effects on early visual perceptual processing. Attention, Perception, Psychophys 2018, 80:929-950.

This paper presents intriguing evidence for the modulation of very early contrast-based perception by cognitive load. It is thus relevant for broader debates about the psychological impact of cognitive load as well as potentially questions about the reach of 'top-down effects' from high-level cognition to early vision.

50. Wang Z, Duff BRL: All Loads Are Not Equal: Distinct Influences of Perceptual Load and Cognitive Load on Peripheral Ad Processing. Media Psychol 2016, 19:589-613.

51. Murphy G, Greene CM: Load Theory behind the Wheel; Perceptual and Cognitive Load Effects. Can J Exp Psychol 2017, 71:191-202.

52. Bengson JJ, Mangun GR: Spatial attention and feature-based attention are differentially sensitive to individual working memory capacity and perceptual load. Vis cogn 2018, 26:545-551.

53. Oksama L, Hyönä J: Position tracking and identity tracking are separate systems: Evidence from eye movements. Cognition 2016, 146:393-409.

54. Mack A, Erol M, Clarke J, Bert J: No iconic memory without attention. Conscious Cogn 2016, 40:1-8.

55. Aru J, Bachmann T: Expectation creates something out of nothing: The role of attention in iconic memory reconsidered. Conscious Cogn 2017, 53:203-210.

56. Fazekas P, Overgaard M: Perceptual consciousness and cognitive access: An 
introduction. Philos Trans R Soc B Biol Sci 2018, 373.

57. Usher M, Bronfman ZZ, Talmor S, Jacobson H, Eitam B: Consciousness without report: Insights from summary statistics and inattention "blindness." Philos Trans R Soc B Biol Sci 2018, 373.

*58. Naccache L: Why and how access consciousness can account for phenomenal consciousness. Philos Trans R Soc B Biol Sci 2018, 373.

59. Overgaard M, Fazekas P: Can No-Report Paradigms Extract True Correlates of Consciousness? Trends Cogn Sci 2016, 20:241-242.

60. Odegaard B, Knight RT, Lau H: Should a few null findings falsify prefrontal theories of conscious perception? J Neurosci 2017, 37:9593-9602.

61. Trübutschek D, Marti S, Ojeda A, King JR, Mi Y, Tsodyks M, Dehaene S: A theory of working memory without consciousness or sustained activity. Elife 2017, 6.

62. Bergström F, Eriksson J: Neural evidence for non-conscious working memory. Cereb Cortex 2018, 28:3217-3228.

*63. Persuh M, Larock E, Berger J: Working memory and consciousness: The current state of play. Front Hum Neurosci 2018, 12:78.

This paper summarises the main evidence thus far for unconscious working memory, and argues that the main evidence attested by defenders of unconscious working memory thus far does not support their claims. However, the authors explicitly remain open to the possibility of unconscious working memory, and suggest how better evidence for this position might be obtained.

64. Stein T, Kaiser D, Hesselmann G: Can working memory be non-conscious? Neurosci Conscious 2016, 2016:niv011.

65. Parr T, Friston KJ: Working memory, attention, and salience in active inference. Sci Rep 2017, 7:14678.

66. Lemaire B, Portrat S: A computational model of working memory integrating timebased decay and interference. Front Psychol 2018, 9.

**67. Oberauer K, Lewandowsky S, Awh E, Brown GDA, Conway A, Cowan N, Donkin C, Farrell S, Hitch GJ, Hurlstone MJ, et al.: Benchmarks for models of short-term and working memory. Psychol Bull 2018, 144:885-958.

This detailed paper features as authors many of the leading figures in contemporary shortterm memory research and proposes a set of broadly theory-neutral benchmarks for shortterm memory research that all leading theories should aim to explain. It provides a wideranging overview of the current state of the field and identifies areas where existing evidence is scanty or absent. In its broadly ecumenical recommendations and detailed summary of existing research, it provides an exceptional resource for both established researchers in the field and those approaching it for the first time.

68. Sherfey JS, Ardid S, Miller EK, Hasselmo ME, Kopell NJ: Prefrontal oscillations modulate the propagation of neuronal activity required for working memory. bioRxiv 2019, doi:10.1101/531574.

69. Lazar M: Working Memory: How Important Is White Matter? Neuroscientist 2017, 23:197-210. 
70. Guida A, Megreya AM, Lavielle-Guida M, Noël Y, Mathy F, van Dijck JP, Abrahamse E: Spatialization in working memory is related to literacy and reading direction: Culture "literarily" directs our thoughts. Cognition 2018, 175:96-100. 